\title{
PRODUCT SEMIGROUPS
}

\author{
E. E. DeVUN
}

(Received 7 April, 1972; revised 12 December 1974)

In earlier work Borrego, Cohen and DeVun (1971a,b) topological semigroups which were uniquely representable in terms of two subsets were investigated. In this note we extend the definition and prove similar results for a larger class of semigroups.

By a $U$-semigroup we mean a semigroup iseomorphic to the unit interval $[0,1]$ with the usual multiplication. A semigroup $S$ is said to be the unique product of $U$-semigroups $S_{1}, S_{2}, \cdots, S_{n}$ if each $S_{i}$ is a $U$-semigroup and for every $\sigma \in G_{n} \quad\left(G_{n}\right.$ is the symmetric group on $n$-elements $) \quad S=$ $S_{\sigma(1)} S_{\sigma(2)} \cdots S_{\sigma(n)}$ and for every non-zero element $s$ of $S$ with $s=$ $x_{\sigma(1)} x_{\sigma(2)} \cdots x_{\sigma(n)}=y_{\sigma(1)} y_{\sigma(2)} \cdots y_{\sigma(n)}$ and $x_{\sigma(i)}, y_{\sigma(i)} \in S_{\sigma(i)}$ we have $x_{\sigma(i)}=y_{\sigma(i)}$. Throughout this paper we will assume all semigroups $S$ have the property that $E(S)$ (the set of all idempotents of the semi-group $S$ ) and $H$ (the union of all the subgroups of $S$ ) consists of $\{0,1\}$ (where 0 is the zero for $S$ and 1 is the identity for $S$ ) and $S$ has no zero divisors.

The author would like to thank the referee for the many helpful comments and suggestions which helped clarify much of the material in this paper.

\section{Unique products and reversibility}

Recall that a semigroup $T$ is left (right) reversible if any two principal right (left) ideals of $T$ intersect. The purpose of this section is to show when semigroups which are the unique product of $U$-semigroups are right or left reversible. Later, in section 3, more results on reversibility are obtained.

LemMA 1.1. If $S$ is the unique product of $U$-semigroups $S_{1}, S_{2}, \cdots, S_{n}$, then $S_{i} S_{j}$ is a subsemigroup of $S$ for all $i \neq j, i, j \in\{1,2, \cdots, n\}$.

Proof. Let $x_{i} \in S_{i}$ and $x_{i} \in S_{j}$. If $x_{j} x_{i}=0$, then $x_{j} x_{i} \in S_{i} S_{j}$. If $x_{j} x_{i} \neq 0$, then for $\sigma \in G_{n}$ with $\sigma(1)=i, \sigma(2)=j$ we can select $y_{\sigma(k)} \in S_{\sigma(k)}, 1 \leqq k \leqq n$, such that

$$
x_{j} x_{i}=y_{\sigma(1)} y_{\sigma(2)} \cdots y_{\sigma(n)}
$$


Now

$$
y_{\sigma(1)} y_{\sigma(2)} \cdots y_{\sigma(n-1)} \neq 0 \text {, }
$$

so for $\delta \in G_{n}$ with $\delta(1)=\sigma(2), \delta(2)=\sigma(1), \delta(k)=\sigma(k), 3 \leqq k \leqq n$, we can pick $z_{\delta(l)} \in S_{\delta(l)}, 1 \leqq l \leqq n$, such that

$$
y_{\sigma(1)} y_{\sigma(2)} \cdots y_{\sigma(n-1)}=z_{\delta(1)} z_{\delta(2)} \cdots z_{\delta(n)}
$$

Hence

$$
x_{i} x_{i}=y_{\sigma(1)} y_{\sigma(2)} \cdots y_{\sigma(n)}=z_{\delta(1)} z_{\delta(2)} \cdots z_{\delta(n)} y_{\sigma(n)} .
$$

Thus $z_{\delta(1)}=x_{j}, z_{\delta(2)}=x_{i}, z_{\delta(l)}=1$, for $3 \leqq l \leqq n-1$ and $z_{\delta(n)} y_{\sigma(n)}=1$ which implies $y_{\sigma(n)}=1$. Continuing this process we obtain $y_{\sigma(l)}=1$ for $3 \leqq l \leqq n$, hence

$$
x_{i} x_{i}=y_{\sigma(1)} y_{\sigma(2)} \in S_{i} S_{j}
$$

This proves $S_{i} S_{i}$ is a subsemigroup of $S$.

Corollary 1.2. Under the hypotheses of 1.1, for $\sigma \in G_{n}$ and $l \in$ $\{1,2, \cdots, n\}, S_{\sigma(1)} S_{\sigma(2)} \cdots S_{\sigma(1)}$ is a subsemigroup of $S$ and is the unique product of $S_{\sigma(1)}, S_{\sigma(2)}, \cdots, S_{\sigma(1)}$.

LEMMA 1.3. If $S$ satisfies the hypotheses of 1.1 , then $S-\{0\}$ is a cancellative subsemigroup of $S$.

Proof. Let $t, u \in S-\{0\}$ and $s \in S_{k}-\{0\}$, where $k$ is any fixed arbitrary integer between 1 and $n$. Suppose $t s=u s$. Then there exist unique representations of $t$ and $u$ as $t_{1} t_{2} \cdots t_{k-1} t_{k+1} \cdots t_{n} t_{k}$ and $u_{1} u_{2} \cdots u_{k-1} u_{k+1} \cdots u_{n} u_{k}$, respectively. From the uniqueness of representation it follows $t_{i}=u_{i}$ for $i \neq k$ and $t_{k} s=u_{k} s$. Since $S_{k}$ is a $U$-semigroup, $t_{k}=u_{k}$, and thus $t=u$. Hence any $s$ coming from a fixed $S_{k}$ can be right cancelled. Now, if $s$ is an arbitrary element of $S-\{0\}$, then let $s$ be represented as $s_{1} s_{2} \cdots s_{n}$. Then, if $t s=u s$, we have $t s_{1} s_{2} \cdots s_{n}=u s_{1} s_{2} \cdots s_{n}$. Set $\hat{t}=t s_{1} s_{2} \cdots s_{n-1}, \hat{u}=u s_{1} s_{2} \cdots s_{n-1}$. Then $\hat{t} s_{n}=\hat{u} s_{n}$, hence $\hat{t}=\hat{u}$, by the above argument. Iterating this process yields $t=u$. This completes the proof.

Definition 1.4. Let $A$ be a subset of an arbitrary semigroup $T$. The normalizer $N(A)$ of $A$ is the set of all $x$ in $T$ with the property that $x A=A x$. If $N(A)=T$, then $A$ is said to be normal in $T$. If $S$ is the unique product of two $U$-semigroups $S_{1}$ and $S_{2}$, then $S_{2}$ normal in $S$ is equivalent to $x S_{2}=S_{2} x$ for all $x \in S_{1}$.

Lemma 1.5. Let $S$ satisfy the hypotheses of 1.1. If $S_{j}$ is normal in $S_{i} S_{j}$ for some $i, j \in\{1,2, \cdots, n\}, j \neq i$, then $S_{k}$ is normal in $S_{i} S_{k}$ or $S_{j}$ is normal in $S_{j} S_{k}$ for $k \in\{1,2, \cdots, n\}$. 
Proof. It was shown in Borrego, Cohen and DeVun (1971b) that for one $U$-semigroup $W$ to be normal in the product of two $U$-semigroups $W V$ we need only show that there exist $v \in V-\{0,1\}, w, w^{\prime} \in W-\{0,1\}$ such that $v w=w^{\prime} v$. Now let $x \in S_{i}, y \in S_{j}$ and $z \in S_{k}$ with none equal to 0 or 1 . Then $x y z=x z_{1} y_{1}=z_{2} x_{1} y_{1}=z_{2} y_{2} x_{1}$ for some $x_{1} \in S_{i}, y_{1}, y_{2} \in S_{j}$, and $z_{1}, z_{2} \in S_{k}$. Also $x y z=y_{3} x z=y_{3} z_{3} x_{2}$ for some $x_{2} \in S_{i}, y_{3} \in S_{i}, z_{3} \in S_{k}$. Hence $x_{1}=x_{2}$. Since $x_{1} y_{1}=y_{2} x_{1}$ and $x y=y_{3} x$ (i.e. $y_{1}$ has no effect on $x_{1}$ and $y$ has no effect on $x$ ), the change in $x$ by $z_{1}$ must be exactly the same as the change in $x$ by $z$. However, this can only occur if $z=z_{1}$ and thus $y z=z y_{1}$ or $z$ and $z_{1}$ have no effect on $x$ and thus $x z=z_{3} x$. This proves the lemma.

In Borrego, Cohen and DeVun (1971b) it was shown that if $S$ is the unique product of two $U$-semigroups, then $S$ is right reversible or left reversible. The following example is due to D. Brown and J. Lawson.

EXAMPLE 1.6. Let $S$ be the one point compactification of the product of the semigroups $R$ and $L$ where

$$
R=\left\{\left(\begin{array}{ll}
x & y \\
0 & 1
\end{array}\right): 0<x \leqq 1, x+y \leqq 1, y \geqq 0\right\}
$$

and

$$
L=\left\{\left(\begin{array}{cc}
x & y \\
0 & 1
\end{array}\right): x \geqq 1, x+y \geqq 1, y \leqq 0\right\},
$$

where $R$ and $L$ have the relative topology of the plane and usual matrix multiplication. Now $S$ is the unique product of four $U$-semigroups but is neither right nor left reversible, since it is the product of a semigroup $R$ which is not left reversible by a semigroup $L$ which is not right reversible see Borrego, Cohen and DeVun (1971b).

THEOREM 1.7. If $S$ is the unique product of $U$-semigroups $S_{1}, S_{2}$ and $S_{3}$, then $T=S-\{0\}$ is either right reversible or left reversible.

Proof. In Borrego, Cohen and DeVun (1971b) it was shown that the subsemigroup $S_{i} S_{j}-\{0\}, i \neq j, i, j \in\{1,2,3\}$ must be iseomorphic to one of the following semigroups.

(a). $\{(x, y): x$ and $y$ are non-negative real numbers $\}$ with the usual addition (the commutative case).

(b). $\left\{\left(\begin{array}{ll}x & y \\ 0 & 1\end{array}\right): x\right.$ and $y$ are real numbers with $x>0, y \geqq 0$, and $\left.x+y \leqq 1\right\}$ with the usual matrix multiplication (right, not left reversible).

(c). $\left\{\left(\begin{array}{ll}x & 0 \\ y & 1\end{array}\right): x\right.$ and $y$ are real numbers with $x>0, y \leqq 0$ and $\left.x+y \leqq 1\right\}$ with the usual matrix multiplication (left not right reversible). 
(d). $\left\{\left(\begin{array}{ll}x & y \\ 0 & 1\end{array}\right): x\right.$ and $y$ are real numbers with $\left.0<x \leqq 1, y \geqq 0\right\}$ with the usual matrix multiplication (both left and right reversible).

(e). $\left\{\left(\begin{array}{ll}x & 0 \\ y & 1\end{array}\right): x\right.$ and $y$ are real numbers with $\left.0<x \leqq 1, y \geqq 0\right\}$ with the usual matrix multiplication (both left and right reversible).

It should be noted that case (d) and (e) are not commutative, but one factor in each case is normal in the semigroup.

A close examination of the possibilities yields two separate cases. Here we distinguish both.

CASE (1). Assume the subsemigroups $S_{1} S_{2}-\{0\}, S_{2} S_{3}-\{0\}$, and $S_{1} S_{3}-$ $\{0\}$ are all left (right) reversible. Let $s, t \in T$ with $s=x y z, t=u v w$ and $x, u \in S_{1}, y, v \in S_{2}, z, w \in S_{3}$ and $x \geqq u$ (where $\geqq$ is the standard cut point order on $[0,1])$. Let $x_{1} \in S_{1}$ with $x x_{1}=u$. Since $S_{1} S_{2}-\{0\}$ and $S_{2} S_{3}-\{0\}$ are left reversible we can select $x_{2}, x_{3} \in S_{1}$ such that $y x_{2}=x_{1} y_{1}$ and $z x_{3}=x_{2} z_{1}$ for some $y_{1} \in S_{2}$ and $z_{1} \in S_{3}$ (see lemma 1, 2, and Borrego, Cohen and DeVun 1971b). Thus

$$
u y_{1} z_{1} T=x x_{1} y_{1} z_{1} T=x y z x_{3} T \subset x y z T,
$$

and $s T \cap t T \neq \varnothing$ if $u y_{1} z_{1} T \cap u v w T \neq \varnothing$. However, $y_{1} z_{1} T \cap v w T \neq \varnothing$, since

$$
y_{1} z_{1}\left(S_{2} S_{3}-\{0\}\right) \cap v w\left(S_{2} S_{3}-\{0\}\right) \neq \varnothing .
$$

So $u y_{1} z_{1} T \cap u v w T \neq \varnothing$, and this completes case 1 .

Case (2). Suppose we have the two-one situation, say $S_{1} S_{2}-\{0\}$ and $S_{2} S_{3}-\{0\}$ are of type c) and $S_{1} S_{3}-\{0\}$ is of type b). Let $s, t \in T$ with $s=x y z$, $t=u v w$ and $x, u \in S_{1}, y, v \in S_{2}, z, w \in S_{3}$ and $x>u$. Let $x_{1} \in S_{1}$, with $x x_{1}=u$. From the characterization of $S_{1} S_{2}-\{0\}$ as being of type c), there exist elements $\hat{x}^{\bullet} \in S_{1}$ and $\hat{y} \in S_{2}$ such that $x_{1} \hat{y}=y \hat{x}$. Hence $x_{1} \hat{y} S_{2} S_{3}=y \hat{x} S_{2} S_{3}$. From the characterization of $S_{1} S_{3}-\{0\}$ as being of type b), there exists $x_{2} \in S_{1}$ with $x \leqq x_{2}$ such that $x_{2} S_{3} \cap z S_{1} \neq \varnothing$. Again appealing to the characterization of $S_{1} S_{2}-\{0\}$, since $x \leqq x_{2}$ there exist $y_{2}, y_{3} \in S_{2}$ such that $\hat{x} y_{3}=y_{2} x_{2}$. From the results of the preceding sentence, pick $x_{4} \in S_{1}$ and $z_{4} \in S_{3}$ such that $x_{2} z_{4}=z x_{4}$. Then $y_{4} z x_{4}=y_{2} x_{2} z_{4}=\hat{x} y_{3} z_{4}$. Since $S_{2} S_{3}-\{0\}$ is of type c), for some real number $\alpha>1, y_{2} z=z^{\alpha} y_{5}=z z^{\alpha-1} y_{5}$, so that $z\left(z^{\alpha-1} y_{5} x_{4}\right)=\hat{x} y_{3} z_{4}$. Let $t=$ $z^{\alpha-1} y_{5} x_{4}$. Then

$$
y z t=y \hat{x} y_{3} z_{4} \in y \hat{x} S_{2} S_{3}=x_{1} \hat{y} S_{2} S_{3}
$$

Therefore

$$
x y z t \in x x_{1} \hat{y} S_{2} S_{3}=u \hat{y} S_{2} S_{3} \subset u S_{2} S_{3} .
$$


Hence there exist $y_{6} \in S_{2}$ and $z_{6} \in S_{3}$ such that $x y z t=u y_{6} z_{6}$. Hence $u y_{6} z_{6}\left(S_{2} S_{3}-\{0\}\right) \subset x y z T$. Now since $S_{2} S_{3}-\{0\}$ is left reversible, we have that $y_{6} z_{6}\left(S_{2} S_{3}-\{0\}\right) \cap v w\left(S_{2} S_{3}-\{0\}\right) \neq \varnothing$. It follows next that $u y_{6} z_{6}\left(S_{2} S_{3}-\{0\}\right) \cap$ $u v w\left(S_{2} S_{3}-\{0\}\right) \neq \varnothing$. Hence $u v w T \cap x y z T \neq \varnothing$. This completes the proof.

According to Brown and Friedberg (1971) if $W$ is a semigroup defined on a closed subset of Euclidean $n$-space $E^{n}$ having non-empty interior in $E^{n}$ and is left (right) reversible, cancellative, and translation functions are homeomorphisms into, then $W$ is embedded in a Lie group. The semigroup $T$ satisfies this, thus is embedded in a Lie group.

COROLLARY 1.8. If $S$ satisfies the hypotheses of 1.7 , then $T=(S-\{0\})$ is embedded in a Lie group.

\section{Uniquely divisible semigroups with two-cells}

Recall a semigroup $S$ is uniquely divisible if for each $s \in S$ and every positive integer $n$ there exists a unique $z \in S$ such that $z^{n}=s$. Borrego, Cohen and DeVun (1971a) showed that if $S$ is a uniquely divisible semigroup on the two-cell with $E(S)=\{0,1\}$, then $S$ is the unique product of two $U$-semigroups, and in their second paper (1971b) a characterization of these semigroups was given. In this section we extend these results.

Definition 2.1. Let $S$ be a uniquely divisible semigroup. Then $[x]$ denotes $\left\{x^{r}: r \text { is a positive rational number }\right\}^{*}$ (where ${ }^{*}$ denotes topological closure), see Hildebrant (1967).

THEOREM 2.2. Let $S$ be a compact uniquely divisible semigroup. If $x, y, z \in$ $S-\{0\}$ with $[x][y]=[y][x]$ and $[x][z]=[z][x]$, then $x y=x z$ implies $y=z$.

Proof. The theorem is clear if $x=1, y=1$, or $z=1$. So we will assume $x, y, z \in S-\{0,1\}$. From Borrego, Cohen and DeVun (1971a) we get the semigroups $[x][y]$ and $[x][z]$ are the unique products of two $U$-semigroups $[x],[y]$ and $[x],[z]$ respectively. Thus we can find $x_{n}, x_{n}^{\prime}, x^{\prime} \in[x], y_{n} \in[y]$, and $z_{n}^{\prime}, z^{\prime} \in[z]$ such that $x_{n} y_{n}=(x y)^{1 / n}=(x z)^{1 / n}=x_{n}^{\prime} z_{n}^{\prime}$ and $x z=z^{\prime} x^{\prime}$. Since $(x y)^{1 / n} \rightarrow 1$, Hudson (1959), we can select a positive integer $k$ such that $(x y)^{1 / k}=x_{k} y_{k}$ and $x_{k} \geqq x^{\prime}$. Let $\bar{x}$ be the unique element of $[x]$ such that $\bar{x} x_{k}=x^{\prime}$. Now

$$
x y y_{k}=x z y_{k}=z^{\prime} x^{\prime} y_{k}=z^{\prime} \bar{x} x_{k} y_{k}=z^{\prime} \bar{x} x_{k}^{\prime} z_{k}^{\prime}=\hat{x} \hat{z}
$$

for some $\hat{x} \in[x]$ and $\hat{z} \in[z]$. Moreover, since $\left[x y y_{k}\right]$ and $[x z]$ are $U$ semigroups $\left(x y y_{k}\right)^{\alpha}=(\hat{x} \hat{z})^{\alpha}$ for $\alpha$ any positive real number. Since a characterization of the semigroup of $[x][y]$ is known Borrego, Cohen and DeVun (1971b) we know we can pick a positive real number $\beta$ such that $\left(x y y_{k}\right)^{\beta}=\tilde{x} y$ 
for some $\tilde{x} \in[x]$ with $\tilde{x}>x$. Now $\tilde{x} y=\left(x y y_{k}\right)^{\beta}=(\tilde{x} \tilde{z})^{\beta}=x^{\prime \prime} z^{\prime \prime}$ for some $x^{\prime \prime} \in[x]$ and $z^{\prime \prime} \in[z]$. Let $u \in[x]$ with $u \tilde{x}=x$. Then

$$
x z=x y=u \tilde{x} y=u x^{\prime \prime} z^{\prime \prime} \in[x][z] .
$$

By the uniqueness of representation of the subsemigroup $[x][z]$ we get $z^{\prime \prime}=z$ and $u x^{\prime \prime}=x=u \tilde{x}$, hence $x^{\prime \prime}=\tilde{x}$. Thus $\tilde{x} y=\tilde{x} z$ with $\tilde{x}>x$. Thus $y=z$.

COROLlARY 2.3. If $S$ is a compact uniquely divisible semigroup and for all $x, y \in S,[x][y]=[y][x]$, then $T=S-\{0\}$ is cancellative.

The following example shows that there are compact uniquely divisible semigroups $S$ with $E(S)=\{0,1\}=H$ and $T=S-\{0\}$ cancellative, but $[x][y] \neq[y][x]$ for some $x, y \in S$. Another example having this property has been given by Hinman (1971).

EXAMPLE 2.4. Let $S$ be the one point compactification of the product of the semigroups $R$ and $U$ where $R=\left\{\left(\begin{array}{ll}a & b \\ 0 & 1\end{array}\right): 0<a \leqq 1, a+b \leqq 1, b \geqq 0\right\}$ with the relative topology of the plane and usual matrix multiplication and $U=(0,1]$ with the usual topology and multiplication. Then $S$ is a compact uniquely divisible semigroup with $E(S)=\{0,1\}=H$ and $T=S-\{0\}$ çancellative, but $[x][y] \neq[y][x]$ when

$$
x=\left(\left(\begin{array}{ll}
\frac{1}{2} & 0 \\
0 & 1
\end{array}\right), \frac{1}{2}\right) \text { and } y=\left(\left(\begin{array}{cc}
\frac{1}{2} & \frac{1}{2} \\
0 & 1
\end{array}\right), 1\right) .
$$

LEMMA 2.5. If $S$ satisfies the conditions of 2.3 , then if $x, y, w \in S-\{0,1\}$ and $[x][y]-\{0\}$ is left reversible and not right reversible, then $[x][w]-\{0\}$ is left reversible.

Proof. Suppose $[x][w]-\{0\}$ is right reversible and not left reversible. Then for every $x_{1} \in[x]-\{0,1\}, w_{1} \in w-\{0,1\}$ there exist $x_{2} \in[x]-\{0,1\}$, $w_{2} \in[w]-\{0,1\}$ with $w_{2}>w_{1}, x_{1}>x_{2}$, and $x_{1} w_{1}=w_{2} x_{2}$. However, for each $x_{1} \in[x]-\{0,1\}, y_{1} \in[y]-\{0,1\}$ there exist $x_{3} \in[x]-\{0,1\}, y_{2} \in[y]-\{0,1\}$ with $y_{1}>y_{2}, x_{3}>x_{1}$ and $x_{1} y_{1}=y_{2} x_{3}$. From the hypothesis $[x][y w]=[y w][x]$, so $x(y w)=\left(y^{\prime} w^{\prime}\right) \tilde{x}$ for some $\left(y^{\prime} w^{\prime}\right) \in[y w]$ and $\tilde{x} \in[x]$. But it is impossible for $y^{\prime} w^{\prime} \in[y w]$ with $y>y^{\prime}$ and $w^{\prime}>w$. Thus $[x][w]-\{0\}$ is left reversible.

The above argument shows $w>w^{\prime}$. Thus it is impossible to have elements $p, q \in S$ with the property that $[p][q]$ is right reversible and not left reversible.

THEOREM 2.6. If $S$ satisfies the hypothesis of 2.3 , then $T=S-\{0\}$ is right reversible or left reversible. 
Proof. From 2.5 we may assume the semigroup $[x][y]-\{0\}$ is left reversible for all $x, y \in T$. Let $w, z \in T$. Then $w([w][z]-\{0\}) \cap$ $z([w][z]-\{0\}) \neq \varnothing$. Thus $w T \cap z T \neq \varnothing$. So $T$ is left reversible.

Thus one can see that if $T=S-\{0\}$ and $S$ satisfies the hypotheses of 2.6 and appropriate Euclidian conditions, then $T$ is embedded in a Lie group.

\section{Reversibility in unique products}

In this section we shall show that if $S$ is the unique product of $U$-semigroups $S_{1}, S_{2}, \cdots, S_{n}$ where $S_{i} S_{j}-\{0\}$ is left (right) reversible, then $T=S-\{0\}$ is left (right) reversible and $S$ is uniquely divisible. The key to this problem lies in the solution for $n=3$. By direct computations we obtained the following results for $n=3$. The calculations concerning (2) can be found in DeVun (to appear).

Let $S$ be the unique product of $U$-semigroups $S_{1}, S_{2}$ and $S_{3}$.

(1). If $S_{2}$ is normal in $S_{1} S_{2}$ and $S_{2}$ is not normal in $S_{2} S_{3}$, then we must have $S_{1}$ normal in $S_{1} S_{2}\left(S_{1} S_{2}\right.$ is commutative), and $S_{3}$ normal in $S_{1} S_{3}$.

(2). If $S_{1} S_{2}-\{0\}, S_{2} S_{3}-\{0\}$ and $S_{1} S_{3}-\{0\}$ are all left (right) reversible and not right (left) reversible, then $\left[s_{1} s_{2}\right]\left[s_{3}\right]=\left[s_{3}\right]\left[s_{1} s_{2}\right]$ for $s_{1} \in S_{1}, s_{2} \in S_{2}$, and $s_{3} \in S_{3}$.

THEOREM 3.1. If $S$ is the unique product of $U$-semigroups $S_{1}, S_{2}, \cdots, S_{n}$ and $S_{i} S_{j}-\{0\}$ left (right) reversible for $i \neq j, i, j \in\{1,2, \cdots, n\}$, then so is $T=S-\{0\}$.

Proof. By assumption the result holds for $k=2$. Assume the result holds for $k=n-1$. We will show the result holds for $k=n$. Let $s, t \in T$ with $s=s_{1} s_{2} \cdots s_{n}, t=t_{1} t_{2} \cdots t_{n}$ with $s_{i}, t_{i} \in S_{i}$ for $i \in\{1,2, \cdots, n\}$ and $t_{1} \leqq s_{1}$. For each $w_{i} \in S_{i}$ and $z_{j} \in S_{j}$ there exist $w_{i}^{\prime} \in S_{i}$ and $z_{j}^{\prime} \in S_{j}$ such that $w_{i} z_{j}=z_{j}^{\prime} w_{i}^{\prime}$. Since $z_{j} \rightarrow 0$ implies $z_{j}^{\prime} \rightarrow 0$, by the continuity of multiplication we can select $z_{1} \in S_{1}$ such that $s z_{1}=t_{1} u_{2}^{\prime} u_{3}^{\prime} \cdots u_{n}^{\prime}$ with $u_{i}^{\prime} \in S_{i}$. Hence

$$
s T \cap t T \supset s_{1} T \cap t T=t_{1} u_{2}^{\prime} u_{3}^{\prime} \cdots u_{n}^{\prime} T \cap t_{1} t_{2} \cdots t_{n} T \neq \varnothing
$$

if $u_{2}^{\prime} u_{3}^{\prime} \cdots u_{n}^{\prime} T \cap t_{2} t_{3} \cdots t_{n} T \neq \varnothing$. However,

$$
\begin{gathered}
u_{2}^{\prime} u_{3}^{\prime} \cdots u_{n}^{\prime} T \cap t_{2} t_{3} \cdots t_{n} T \supset u_{2}^{\prime} u_{3}^{\prime \prime} \cdots u_{n}^{\prime}\left(S_{2} S_{3} \cdots S_{n}-\{0\}\right) \\
\cap t_{2} t_{3} \cdots t_{n}\left(S_{2} S_{3} \cdots S_{n}-\{0\}\right) \neq \varnothing .
\end{gathered}
$$

So our results hold.

Note that under the conditions of 3.1, $T$ is embedded in a Lie group.

THEOREM 3.2. If $S$ satisfies the conditions of 3.1, then $S$ is uniquely divisible. 
Proof. In Borrego, Cohen and DeVun (1971b) the result was shown for $k=2$. Assume the result holds for $(n-1)$ products. Now $S$ is clearly divisible since the map $s \rightarrow s^{n}$ sends the boundary of $S$ onto the boundary of $S$. To show $S$ is uniquely divisible we distinguish three cases.

CASE 1. Suppose $S_{i} S_{j}-\{0\}$ is left reversible and not right reversible for each pair $i \neq j, i, j \in\{1,2, \cdots, n\}$. Let $s, t \in S-\{0\}$ with $s^{m}=t^{m}$ for some positive integer $m$, and $s=s_{1} s_{2} \cdots s_{n}, t=t_{1} t_{2} \cdots t_{n}$ with $s_{i}, t_{i} \in S_{i}$, $i \in\{1,2, \cdots, n\}$. From the discussion preceding 3.1 we get

$$
s^{m}=s_{1}\left(s_{2} s_{3} \cdots s_{n}\right) \cdots s_{1}\left(s_{2} s_{3} \cdots s_{n}\right)=s_{1}^{p}\left(s_{2} s_{3} \cdots s_{n}\right)^{q},
$$

and

$$
t^{m}=t_{1}\left(t_{2} t_{3} \cdots t_{n}\right) \cdots t_{1}\left(t_{2} t_{3} \cdots t_{n}\right)=t_{1}^{r}\left(t_{2} t_{3} \cdots t_{n}\right)^{u}
$$

Thus $s_{1}^{p}=t_{1}^{r}$ and $\left(s_{2} s_{3} \cdots s_{n}\right)^{q}=\left(t_{2} t_{3} \cdots t_{n}\right)^{\mu}$. Thus $s_{1}=t_{1}^{r / p}$ and $s_{2} s_{3} \cdots s_{n}=$ $\left(t_{2} t_{3} \cdots t_{n}\right)^{u / q}$. So $s, t \in\left[s_{1}\right]\left[s_{2} s_{3} \cdots s_{n}\right]=\left[t_{1}\right]\left[t_{2} t_{3} \cdots t_{n}\right]$ with $s^{m}=t^{m}$. But $\left[s_{1}\right]\left[s_{2} s_{3} \cdots s_{n}\right]$ is uniquely divisible Borrego, Cohen and DeVun (1971b), and we get $s=t$.

CASE 2. Suppose there exists $i, j \in\{1,2, \cdots, n\} i \neq j$ with $S_{j}$ is normal in $S_{i} S_{i}$ but $S_{i}$ does not commute with $S_{j}$. From the discussion preceding 3.1 we have for each $k \in\{1,2, \cdots, n\}, S_{j}$ is normal in $S_{j} S_{k}$. Thus for $k \in\{1,2, \cdots, n\}$ with $s_{k} \in S_{k}$ and $s_{j} \in S_{j}$ there exists $s_{j}^{\prime} \in S_{j}$ such that $s_{k} s_{j}=s_{j}^{\prime} s_{k}$. Now let $s, t \in S-\{0\}$ with $s^{m}=t^{m}$. Set $s=s_{1} s_{2} \cdots s_{n}$ and $t=t_{1} t_{2} \cdots t_{n}$ with $s_{i}, t_{i} \in S_{i}$, $i \in\{1,2, \cdots, n\}$. Then

$$
\begin{aligned}
s^{m} & =\left(s_{1} s_{2} \cdots s_{n-1} s_{j} s_{j+1} \cdots s_{n}\right) \cdots\left(s_{1} s_{2} \cdots s_{i-1} s_{j} s_{j+1} \cdots s_{n}\right) \\
& =s_{i}^{\alpha}\left(s_{1} s_{2} \cdots s_{i-1} s_{j+1} \cdots s_{n}\right)^{m}
\end{aligned}
$$

and

$$
\begin{aligned}
t^{m} & =\left(t_{1} t_{2} \cdots t_{j-1} t_{j} t_{j+1} \cdots t_{n}\right) \cdots\left(t_{1} t_{2} \cdots t_{j-1} t_{j} t_{j+1} \cdots t_{n}\right) \\
& =t_{j}^{\beta}\left(t_{1} t_{2} \cdots t_{j-1} t_{j+1} \cdots t_{n}\right)^{m}
\end{aligned}
$$

for appropriate real numbers $\alpha$ and $\beta$. Since

$$
\left(s_{1} s_{2} \cdots s_{j-1} s_{j+1} \cdots s_{n}\right)^{m}=\left(t_{1} t_{2} \cdots t_{j-1} t_{j+1} \cdots t_{n}\right)^{m}
$$

we get

$$
s_{l} s_{2} \cdots s_{j-1} s_{j+1} \cdots s_{n}=t_{1} t_{2} \cdots t_{j-1} t_{j+1} \cdots t_{n} .
$$

However, this implies $s_{j}=t_{j}$. So $s=t$.

Case 3. Suppose there exists $i, j \in\{1,2, \cdots, n\}, i \neq j$ with $S_{i}$ commuting with $S_{j}$. Then for each $k \in\{1,2, \cdots, n\}$ we must have $S_{i}$ commute with $S_{k}$ or $S_{j}$ commutes with $S_{k}$. Let $S_{i 1}, S_{i 2}, \cdots, S_{i k}$ commute with $S_{i}$ alone, $S_{j 1}, S_{j 2} \cdots S_{j l}$ commute with $S_{j}$ alone, and $S_{q 1}, S_{q 2}, \cdots, S_{q r}$ commute with both $S_{i}$ and $S_{j}$. 
Using the results of (1) and lemma 1.5 several times one sees that for all $t \in\{1,2, \cdots, r\} S_{q r}$ commutes with $S_{i u}$ and $S_{j v}$ for all $u \in\{1,2, \cdots, k\}$ and $v \in\{1,2, \cdots, l\}$. Since $S_{q t}$ commutes with $S_{i}\left(S_{j}\right)$, and $S_{i}\left(S_{j}\right)$ does not commute $S_{i v}\left(S_{i u}\right), S_{q t}$ must commute with $S_{j v}\left(S_{i u}\right)$. Also $S_{i u}$ commutes with $S_{j v}$ for all $u \in\{1,2, \cdots, k\}$ and $v \in\{1,2, \cdots, l\}$. This can be seen since $S_{i u}$ commutes with $S_{i}, S_{i v}$ does not commute with $S_{i}$, so $S_{i u}$ must commute with $S_{i v}$. Now let $s, t \in S-\{0\}$ with $s^{m}=t^{m}$ and

$$
s=s_{i}\left(s_{11}, s_{i 2}, \cdots, s_{j k}\right) s_{i}\left(s_{i 1} s_{i 2} \cdots s_{i l}\right)\left(s_{q 1} s_{q 2} \cdots s_{q 1}\right)
$$

and

$$
t=t_{i}\left(t_{i 1} t_{j 2} \cdots t_{i k}\right) t_{j}\left(t_{i 1} t_{i 2} \cdots t_{i l}\right)\left(t_{q 1} t_{q 2} \cdots t_{q 1}\right)
$$

$s_{i}, t_{i} \in S_{i} ; s_{j v}, t_{j v} \in S_{j v} ; s_{j}, t_{j} \in S_{j} ; s_{i u}, t_{i u} \in S_{i u} ; s_{q u}, t_{q r} \in S_{q u}$. Then

$$
\begin{aligned}
s^{m} & =\left(s_{i}\left(s_{j 1} s_{j 2} \cdots s_{i k}\right)\right)^{m}\left(s_{j}\left(s_{i 1} s_{i 2} \cdots s_{i l}\right)\right)^{m}\left(s_{q 1} s_{q 2} \cdots s_{q v}\right)^{m} \\
& =\left(t_{i}\left(t_{j 1} t_{j 2} \cdots t_{j k}\right)\right)^{m}\left(t_{j}\left(t_{i 1} t_{i 2} \cdots t_{i l}\right)\right)^{m}\left(t_{q 1} t_{q 2} \cdots t_{q v}\right)^{m} .
\end{aligned}
$$

Since each of the components is uniquely divisible $s=t$. This completes the proof.

Finally, it can be seen in Brown and Friedburg (1968) that $S$ may be a commutative uniquely divisible semigroup without being a unique product. Thus the converse of 3.2 is false.

\section{References}

J. T. Borrego, H. Cohen, and E. E. DeVun (1971a), 'Uniquely representable semigroups on the two-cell', Pacific J. Math. 38, No. 3.

J. T. Borrego, H. Cohen, and E. E. DeVun (1971b), 'Uniquely representable semigroups II', Pacific J. Math. 39, No. 2.

D. F. Brown and Michael Friedberg (1968), 'Representation theorems for uniquely divisible semigroups', Duke Math. J. 35, 341-352.

D. R. Brown and Michael Friedberg (1971), 'Linear representations of certain compact semigroups', Trans. Amer. Math. Soc. 160, 453-465.

E. E. DeVun, 'Semigroups with two-cells', Protugaliae Mathematica, (submitted for publication).

J. A. Hildebrant (1967), 'On compact unithetic semigroups', Pacific J. Math. 21, 265-273.

B. F. Hinman (1971), 'Products of threads in topological semigroups', Dissertation, University of Houston, Houston, Texas.

A. L. Hudson (1959), 'Some semigroups on the two-cell', Proc. Amer. Math. Soc. 10, 648-655.

Department of Mathematics,

Wichita State University,

Kansas 67208,

U.S.A. 